\title{
CONSULTA DE ENFERMAGEM A GESTANTES COM ANEMIA FERROPRIVA*
}

\author{
Sonia Maria Oliveira de Barros** \\ Cláudia Aparecida Ribeiro Costa***
}

BARROS, S.M.O.; COSTA, C.A.R. Consulta de enfermagem a gestantes com anemia ferropriva. Rev.latinoam.enfermagem, Ribeirão Preto, v. 7, n. 4, p. 105-111, outubro 1999.

Este trabalho teve como objetivos: construir um protocolo de levantamento de dados e diagnósticos de enfermagem, e conhecer os diagnósticos de enfermagem mais freqüentes. O protocolo construído foi aplicado em 52 consultas de enfermagem para as gestantes que apresentavam valores de hemoglobina menores que 11,0g/dl. Os diagnósticos de enfermagem mais freqüentes foram: nutrição alterada, risco para infecção, manutenção do lar prejudicada; déficit de conhecimento sobre a alimentação, risco para lesão fetal relacionada a diminuição da perfusão útero-placentária.; risco de não-comprometimento. A partir dos diagnósticos de enfermagem foram planejadas as intervenções e os resultados esperados com a sua aplicação.

UNITERMOS: anemia hipocrômica, enfermagem obstétrica, cuidado pré-natal, diagnóstico de enfermagem

\section{INTRODUÇÃO}

A adequada quantidade de ferro é essencial para o bem-estar materno e para o crescimento fetal. No entanto, a anemia é um dos problemas médicos mais encontrados na gravidez (BARGER \& FEIN, 1997).

A anemia pode ser definida como uma concentração sangüínea de hemoglobina abaixo dos valores de normalidade para determinada faixa etária e sexo (LIMA et al., 1994). Quando ocorre durante a gestação, dependendo da gravidade, pode ter efeito deletério para a mãe e para o feto.

Em 1968, o Grupo Científico sobre Anemias Nutricionais da Organização Mundial da Saúde (OMS, 1968) considerou a concentração de hemoglobina de 11,0 $\mathrm{g} / \mathrm{dl}$ como limite inferior aceitável para a gestante. ROODENBURG (1995) e outros autores aceitam estes valores, outros têm sugerido o valor de $10,4 \mathrm{~g} / \mathrm{dl}$ como mais apropriado.

A assistência de enfermagem a gestantes vem sendo realizada há cerca de trinta anos pelas enfermeiras obstetras da Disciplina Enfermagem Obstétrica, em uma maternidade conveniada a Universidade Federal de São Paulo. Nesse período, a anemia ferropriva foi uma das complicações mais freqüentes na assistência de enfermagem obstétrica. Esta observação levou a realização de trabalhos científicos sobre a anemia ferropriva como fator de risco obstétrico e a suas repercussões na gestação (CALLEGHARI et al., 1994; COSTA \& BARROS, 1996), tendo em vista que, a anemia ferropriva não implica em encaminhamento ao serviço de pré-natal de alto risco, continuando a assistência sobre a responsabilidade das enfermeiras obstetras.

De acordo com COSTA \& BARROS (1996) a anemia ferropriva esteve presente em $45,0 \%$ das gestantes assistidas por enfermeiras obstetras no serviço acima mencionado. Esta prevalência levou as autoras a elaborarem um protocolo de orientações de enfermagem para prevenção e tratamento da anemia ferropriva na gestação e a sugerirem a necessidade de assistência direcionada para estas gestantes com realização de consultas de enfermagem.

Isto porque, na consulta de enfermagem o enfermeiro utiliza componentes do método científico para identificar situações de saúde/doença, prescrever e implementar medidas de enfermagem que contribuam para a promoção, prevenção, proteção da saúde, recuperação e reabilitação do indivíduo, família e comunidade (COREN, 1997). As consultas de enfermagem específicas a esse grupo de gestantes teriam, também, as seguintes metas:

- que as gestantes completem a gravidez e o parto sem as complicações relacionadas a anemia ferropriva;

- que os níveis de hemoglobina retornem aos valores normais;

- que as gestantes compreendam o tratamento prescrito.

\footnotetext{
* Trabalho subsidiado pelo PIBIC/CNPq/UNIFESP/EPM

** Enfermeira. Doutora em Enfermagem Materna e Infantil. Professora Adjunto da Disciplina Enfermagem Obstétrica do Departamento de Enfermagem da UNIFESP/EPM

*** Enfermeira. Aluna do Curso de Especialização em Enfermagem Obstétrica da UNIFESP/EPM. Bolsista PIBIC/CNPq 1996 e 1997
} 
Para que pudéssemos atingir estas metas, realizamos este trabalho, com os seguintes objetivos:

1. Construir um protocolo de levantamento de dados e diagnósticos de enfermagem a ser utilizado na consulta de enfermagem a gestantes com anemia ferropriva.

2. Identificar os diagnósticos de enfermagem mais freqüentes entre essas gestantes e propor as intervenções de enfermagem.

\section{MATERIAL E MÉTODO}

- Local: o trabalho foi desenvolvido no serviço pré-natal de uma maternidade conveniada a Universidade Federal de São Paulo, na qual a Disciplina Enfermagem Obstétrica é responsável pela assistência às gestantes, após o projeto ter sido aprovado em reunião científica e pela Comissão de Ética em Pesquisa da universidade.

- População e critério de inclusão: foram realizadas 52 consultas de enfermagem às gestantes que apresentaram valores de hemoglobina iguais ou inferiores a $11,0 \mathrm{~g} / \mathrm{dl}$. - Como referencial metodológico utilizamos os padrões de respostas humanas da North American Nursing Diagnosis Association (NANDA, 1996).

\section{RESULTADOS E DISCUSSÃO}

O foco central da enfermagem é o cuidado do cliente. Os aspectos humanísticos e científicos do cuidado de enfermagem são executados através do processo de enfermagem. Portanto, o processo de enfermagem é o método científico de identificar e resolver problemas de enfermagem (GORDON, 1994) sendo composto pelas seguintes etapas: levantamento de dados, diagnósticos de enfermagem, planejamento de intervenções e avaliação.

O levantamento sistemático dos dados das clientes é o primeiro passo do processo de enfermagem, e presta-se para determinar o estado de saúde atual e passado da cliente, seu estado funcional e para a avaliação do seu padrão de resolução de problemas presentes e passados (CARPENITO, 1997). Os dados podem ser obtidos através de entrevista, exame físico, observação, revisão dos registros e relatos de diagnósticos.

Muito mais que um banco de informações clínicas, o protocolo de levantamento de dados deve ser centrado nos aspectos bio-psico-espirituais, de forma a conduzir a enfermeira a atender adequadamente as necessidades individuais. De acordo com GALLANI et al. (1996) quando a enfermeira proporciona ao paciente uma participação ativa no seu atendimento, escutando-o com interesse, disposição e favorecendo a sua liberdade de expressão verbal, consegue percebê-lo como um ser único e, dessa forma, planejar a assistência de enfermagem individualizada.
Para o estabelecimento de uma relação benéfica entre a enfermeira e a cliente, é necessário que a entrevista inicial seja bem conduzida e que haja uma boa comunicação no relacionamento (DOENGES \& MOORHOUSE, 1992).

A elaboração do protocolo de levantamento de dados e diagnósticos de enfermagem (assessment) foi baseada na prática clínica das autoras e em amplo levantamento bibliográfico sobre a anemia ferropriva na gestação de forma que o protocolo pudesse contemplar os aspectos gerais e permitisse focalizar aspectos relevantes para a assistência de enfermagem específica. Foram consultados trabalhos nacionais de enfermeiros que elaboraram protocolos de levantamento de dados em seus serviços (MARIA, 1990; SILVA, 1995; GALLANI et al., 1996; HASSMAN, 1996), e realizadas consultas a especialistas em diagnósticos de enfermagem.

Optamos por compor o protocolo pelos dados básicos iniciais a serem preenchidos na primeira consulta da gestante, de forma a permitir a elaboração de inferências pré-diagnósticas amplas, e organizar de acordo com os padrões de respostas humanas da NANDA (1996).

Iniciamos com o padrão conhecer, de forma a proporcionar condições para que a gestante verbalizasse o seu conhecimento sobre a anemia ferropriva, a gestação e as implicações da ocorrência da anemia durante a gravidez.

Nos itens seguintes, foram abordados os dados fisiológicos e, também, os processos que envolvem as considerações psicológicas, sócioculturais, desenvolvimentais e espirituais. Segundo CARPENITO (1997), a enfermeira necessita compreender os padrões de interação entre as áreas acima citadas para identificar os pontos fortes e as limitações da cliente e auxiliá-la a alcançar a saúde ideal.

A necessidade de ferro durante a gestação é de 4 mg por dia, aumentando para 6 a $8 \mathrm{mg}$ próximo ao termo (BARGER \& FEIN, 1997). A anemia por deficiência de ferro representa $90,0 \%$ das anemias que podem ocorrer durante a gravidez, isto porque os hábitos alimentares da gestante brasileira geralmente não fornece a necessária reposição de ferro.

A deficiência de ferro durante a gestação pode ser assintomática, entretanto a gestante tem aumento do risco de desenvolver infecções, fadiga e hemorragia pósparto. E o feto tem maior chance de apresentar prematuridade, baixo peso ao nascer, natimortalidade e morte neonatal (BARGER \& FEIN, 1997).

O protocolo de levantamento de dados e diagnósticos de enfermagem foi utilizado em 52 consultas de enfermagem a gestantes com anemia ferropriva, e os diagnósticos de enfermagem mais freqüentes foram:

- Nutrição alterada: ingesta menor que as necessidades corporais, relacionada a verbalização de falta de alimentos. 
- Nutrição alterada: ingesta maior que as necessidades corporais, relacionada a falta de conhecimento nutricional básico.

- Risco para infecção relacionado a defesa secundária insuficiente pela diminuição da hemoglobina.

- Manutenção do lar prejudicada relacionada a falta de recursos financeiros suficientes.

- Déficit de conhecimento sobre a alimentação adequada durante a gestação.

- Risco para lesão fetal relacionado a diminuição da perfusão útero-placentária.

- Risco para não comprometimento relacionado a dieta alimentar recomendada para o tratamento.

Ressaltamos que o plano de cuidados de enfermagem para a gestante com anemia ferropriva é individualizado, deve basear-se nos diagnósticos de enfermagem encontrados, mas deve também, ter os seguintes objetivos: prevenir as complicações relacionadas a anemia ferropriva, que os valores de hemoglobina retornem aos níveis normais, que o crescimento e desenvolvimento fetal não apresente as alterações relacionadas a anemia materna e que o tratamento prescrito seja realizado.

Desde a prevenção até o tratamento da anemia, o objetivo essencial do cuidado de enfermagem deve ser o aconselhamento sobre a dieta alimentar adequada.

A gestante deve ser informada sobre as dietas variadas que proporcionam a ingesta e absorção adequada de ferro, e sobre a importância da suplementação férrica. Os protocolos de orientação de enfermagem são muito importantes no cuidado pré-natal porque auxiliam a enfermeira a programar a assistência individualizada focalizando os pontos relevantes da alimentação. Os protocolos de orientação de enfermagem focalizados na gestante com anemia ferropriva, proporcionam melhores resultados tanto na prevenção quanto no tratamento desta patologia (COSTA \& BARROS, 1996).

A seguir descreveremos o plano de cuidados de enfermagem que foi elaborado a partir dos diagnósticos de enfermagem encontrados, e aplicado na assistência a gestantes com anemia ferropriva.

Diagnóstico de enfermagem: Nutrição alterada: ingesta menor que as necessidades corporais, relacionada a verbalização de falta de alimentos por barreiras econômicas.

\begin{tabular}{ll}
\multicolumn{1}{c}{$\begin{array}{c}\text { INTER VENÇÕES DE } \\
\text { ENFERMAGEM }\end{array}$} & \multicolumn{1}{c}{$\begin{array}{c}\text { RESULTADOS } \\
\text { ESPERADOS }\end{array}$} \\
\hline $\begin{array}{l}\text { Eliminar ou reduzir os fatores } \\
\text { contribuintes }\end{array}$ & $\begin{array}{l}\text { Que a cliente identifique um } \\
\text { método para obter regularmente } \\
\text { os alimentos }\end{array}$ \\
$\begin{array}{l}\text { Sugerir compras em } \\
\text { Quoperativas verbalize o cons umo de } \\
\text { Encaminhar a programas } \\
\text { governamentais ou serviço } \\
\text { social }\end{array}$ & \\
\hline
\end{tabular}

Diagnóstico de enfermagem: Nutrição alterada: ingesta maior que as necessidades corporais; relacionada a falta de conhecimento nutricional básico.

INTERVENÇÕES DE
ENFERMAGTADOS
ESPERADOS

Planejar com os alimentos que a Que a gestante discuta as gestante refere consumir, dietas necessidades nutricionais, e não ricas em ferro heme e de baixas ultrapasse o peso ideal calorias

Discutir a ingesta nutricional e o Que agestante discuta os efeitos ganho de peso durante agestação do exercício sobre o controle de Revisar coma cliente os itens da peso

dieta alimentar

Recomendar aumento da atividade fisica segundo os limites, disponbilidade e a idade gestacional

Diagnóstico de enfermagem: Risco para infecção relacionado a defesa secundária insuficiente pela acentuada diminuição da hemoglobina durante a gestação.

\section{INTER VENÇÕES DE} ENFERMAGEM

Estimular a suplementação fémica, para melhorar o estado nutricional e aumentar a resistência as infecções

Fazer exame fisico detalhado para detectar possíveis focos de infe cção

Ensinar a importância da higiene corporal e genital para a prevenção das infecções

\section{RESULTADOS ESPERADOS}

Que a gestante verbalize a sua adesão ao tratamento da anemia, a dieta proposta e não apresente infecções

Detectare tratar precoomenteas infecções para preverir danos matemos, fetais e neonatais Que a gestante e seu feto não apresentem infecções

Diagnóstico de enfermagem: Manutenção do lar prejudicada relacionada a falta de recursos financeiros suficientes.

\section{INTER VENÇÕES DE ENFERMAGEM \\ RESULTADOS ESPERADOS}

Encaminhar para serviços de Que a gestante procure os assistência social e seguro serviços e mellhore as condições desemprego, se for necessánio individuais e familiares

Diagnóstico de enfermagem: Déficit de conhecimento sobre a alimentação adequada durante a gestação.

\section{INTERVENÇÕES DE ENFERMAGEM}

Ensinar a importância dos alimentos e a sua ingestão equilibrada

Onentar quanto o consumo de fontes de ácido ascórbico (vitarima C); meia horaantes das refeições associado a suplementação fémica

Evitar chá, café, leite e seus derivados antes e após as refeições

\section{RESULTADOS ESPERADOS}

Que a gestante verbalize a ingestão adequada

Aumentar a absorção de ferro heme 
Diagnóstico de enfermagem: Risco para lesão fetal relacionada a diminuição da perfusão úteroplacentária.

\section{INTER VENÇÕES DE} ENFERMAGEM

Explicar os maleficios do furno $\mathrm{e}$ recomendar que a gestante não furne e, também, evite ambientes fechados com presença de fumantes

Orientar para que não falte nas coletas de material para exames de rotina

\section{RESULTADOS ESPERADOS}

Que agestante evite a exposição ao furno.

Mantervalores de hemoglobina, hematócrito e ferro sérico dentro dos limites de normalidade

Diagnóstico de enfermagem: Risco para nãa comprometimento relacionado a dieta alimentar recomendada para o tratamento.

\section{INTER VENÇÕES DE} ENFERMAGEM

\section{RESULTADOS} ESPERADOS
Se necessário, focar as respostas emocionais que levarn a recusa (por exemplo: ansiedade, depressão, problemas de relacionamento, etc.)

\section{CONSIDERAÇÕES FINAIS}

A enfermeira obstetra como membro atuante na equipe de assistência pré natal deve contribuir efetivamente para a prevenção e o tratamento da anemia na gestação.

$\mathrm{Na}$ consulta de enfermagem às gestantes com anemia ferropriva, o protocolo de levantamento de dados e diagnósticos de enfermagem mostrou-se um instrumento eficaz, facilitou o relacionamento entre a enfermeira e a gestante, a identificação, controle e prevenção da anemia, e proporcionou o planejamento das intervenções de enfermagem individualizadas.

As gestantes foram estimuladas a manter ou restabelecer o nível de saúde. Temos certeza que, dessa forma, as enfermeiras obstetras contribuirão para a diminuição da mortalidade perinatal associada a anemia na gestação e da morte materna nas regiões em desenvolvimento.

\section{NURSING CARE TO PREGNANT WOMEN WITH IRON DEFICIENCY ANEMIA}

This study was carried out with the following objectives: to set up a protocol of data survey and nursing diagnosis, and to detect the most frequent nursing diagnosis among pregnant women with iron deficiency anemia. The protocol was applied in 52 nursing visits to pregnant women who presented hemoglobin values lower than 11.0g/dl.The most frequent nursing diagnosis were: altered nutrition, risk to infection, impaired maintenance of the home; knowledge deficit on adequate feeding; risk to fetal injury regarding decrease in uteroplacental perfucion; non-compliance risk. Based on nursing diagnoses, interventions and nursing expected results with its application were planned.

KEY WORDS: anemia hypochromic, obstetrical prenatal nursing care, nursing diagnosis

\section{CONSULTA DE ENFERMERÍA A MUJERES EMBARAZADAS CON ANEMIA FERROPÉNICA}

Realizamos este trabajo con los siguientes objetivos: construir un protocolo de levantamiento de datos y conocer los diagnósticos de enfermería más frecuentes entre las mujeres embarazadas con anemia ferropénica. El protocolo fue aplicado en 52 consultas de enfermería para las gestantes que presentaban valores de hemoglobina abajo de 11,0 g/dl. Los diagnósticos de enfermería más frecuentes fueron: nutrición alterada, riesgo de infección, manutención del hogar perjudicada, déficit de conocimiento sobre la alimentación, riesgo de lesión fetal relacionada con la disminución de perfusión útero-placentária, riesgo de no comprometimiento. A partir de los diagnósticos de enfermería fueron planeadas las intervenciones y los resultados esperados con su aplicación. 
Anexo

Protocolo de levantamento de dados e diagnósticos de enfermagem em gestantes com anemia ferropriva

Data do atendimento:

NOME IDADE RG

ENDEREÇO

BAIRRO TELEFONE NATURALIDADE

COR ESCOLARIDADE RELIGIÃO

ESTADO CIVIL PROFISSÃO SITUAÇÃO CONJUGAL OCUPAÇÃO

D.U.M. / I Idade gestacional: semanas D.P.P. _ _ / _ _ Data do último UGS / / Idade gestacional semanas DPP / /

VALOR DA HEMOGLOBINA

DATA

VALOR DO FERRO SÉRICO DATA

O que você sabe sobre a anemia na gestação?

Você conhece o tratamento para a anemia na gestação?
( ) Não
( ) $\mathrm{Sim}$

Qual é?

Como a anemia pode interferir no desenvolvimento da sua gravidez?

Você sabe como evitar a anemia?

( ) Não ( ) Sim. Como?

Você está usando alguma medicação para tratamento da anemia nesta gravidez?

( ) Não ( ) Sim. Qual?

Quem recomendou o uso?

Desde quando está usando?

Como você está usando esta medicação?

Via de administração

Em quais horários você está usando as medicações?

Sabe quais são os objetivos do uso destas medicações?

( ) Não ( ) Sim. Quais?

Impressões do entrevistador

Qual a renda familiar mensal da sua família? (em número de salários mínimos)

Você considera a renda familiar suficiente?

( ) Sim ( ) Não. Por quê?

Isso a preocupa? ( ) Sim ( ) Não. Por quê?

A gravidez trouxe algum problema no relacionamento com sua família? ( ) Não ( ) Sim. Por quê?

E com seus amigos? ( ) Não ( ) Sim. Por quê?

Sente-se sozinha? ( ) Não ( ) Sim. Por quê?

A anemia na gravidez prejudicou a sua atividade profissional?

( ) Não ( ) Sim. Como?

Impressões do entrevistador

O que você sentiu ou o que pensou após ficar sabendo que está com anemia?

Você acha que o tratamento da anemia na gravidez vai mudar o seu estilo de vida? ( ) Não ( ) Sim. Por quê?

Você quer fazer o tratamento que lhe for proposto?

( ) Não ( ) Sim. Por quê?

Com quem você mora?

Número de pessoas que moram na sua casa?

Sua moradia é: ( ) própria ( ) alugada ( )

Fica em favela: ( ) Sim ( ) Não
Possui água encanada: ( ) Sim ( ) Não

Você acha que a anemia dificultou as suas atividades?

( ) Sim ( ) Não. Quais atividades?

Por quê?

Você apresenta alguma alteração em relação ao sono?

( ) Não ( ) Sim. Descreva

E quanto as atividades de lazer que você costumava ter, sofreram alterações? ( ) Não ( ) Sim. Descreva

Você pratica esporte(s) ( ) Não. Por quê?

( ) Sim. Qual(is)?__ número de vezes/semana

Você fuma? ( ) Não ( ) Sim. Quantos cigarros por dia?

Durante esta gravidez está fumando? ( ) Mais ( ) Menos ( )

O mesmo número de cigarros ( ) ( ) Parou de fumar

Você costuma tomar bebidas alcoólicas?

( ) Não ( ) Sim número de doses/dia

Usa drogas ilícitas? (Maconha, Cocaína, e outros)

( ) Não ( ) Sim. Quais?

Impressões do entrevistador: (observar autocuidado e higiene corporal)

Qual é a sua religião?

Acha que a sua religião teve alguma influência no fato de você ter anemia? ( ) Não ( ) Sim. Por quê?

Acha que a religião pode ajudá-la neste momento? ( ) Não ( )

Sim. Por quê?

Obervação do entrevistador em relação a alteração de comportamento ou humor

Houve modificações no seu modo de vida ao saber que está com a anemia durante a gestação? ( ) Não ( ) Sim. Quais?

Impressões do entrevistador

Quais são os seus sentimentos em relação as possibilidades da evolução da anemia na gestação?

Impressões do entrevistador

A paciente apresenta:

( ) Comunicação sem alterações

( ) Comunicação prejudicada, por:

( ) Percepção alterada

( ) Déficit auditivo ( ) parcial ( ) total

( ) Déficit visual ( ) parcial ( ) total

( ) Origem cultural

( ) Outros

Impressões do entrevistador

A paciente demonstra: ( ) Excitação ( ) Tagarelice ( ) Nervosismo ( ) Preocupação ( ) Impaciência ( ) Insônia ( ) Olhando ao redor

Nutricional:

Peso atual: $\quad$ Peso antes da gestação Altura

Perímetro do pulso:

Diagnóstico nutricional: ( ) Desnutrida ( ) Eutrófica ( )

Obesa: grau

Você tem horários para se alimentar. ( ) Não ( ) Sim. Quais?

Quais os alimentos que você consome habitualmente nas suas refeições e em quais quantidades?

Dentre os seguintes alimentos quais tem preferência:

( ) Laranja ( ) Banana ( ) Maçã ( ) Limão ( ) Morango

( ) Carne vermelha ( ) Vísceras ( ) Alface

Após cada refeição, quanto tempo permanece sem vontade de ingerir outros alimentos? 
Sente que seu estômago permanece repleto durante muito tempo após as refeições? ( ) Não ( ) Sim. O que sente?

O que faz para melhorar?

Apresenta ou apresentou:

Náuseas: ( ) Não ( ) Sim

Vômitos: ( ) Não ( ) Sim

Quando?

Quanto?

Alimenta-se em resposta a alguma situação externa?

\section{ELIMINAÇÕES}

Qual é sua freqüência de evacuações?

Quantas vezes por dia e/ou semana?

Qual é o aspecto e quantidade?

Faz uso de laxantes?

Qual é a freqüência do uso de laxantes?

Em quais situações faz uso?

Quantas vezes por dia apresenta micção?

Qual é a quantidade e o aspecto?

Apresenta sangramento e/ou leucorréia vaginal?

ANTECEDENTES OBSTÉTRICOS

Antecedentes Obstétricos

Número de gestações Número de parto: normais

cesáreas

Número de abortamentos anteriores: espontâneos

provocados

Número de filhos vivos

Causa(s) do(s) óbito(s)

Teve partos prematuros? Número de filhos mortos

\section{REFERÊNCIAS BIBLIOGRÁFICAS}

01. BARGER, M.K.; FEIN, E. High-risk pregnancy. In: NICHOLS, F.H.; ZWELLING, E. Maternal newborn nursing: theory and practice. New York: Saunders, 1997. Cap.21, p.675-78.

02. CALLEGHARI, A H.; BARROS, S.M.O.; MARIN, H.F.; DORCAS, A identificação de fatores de risco gravídico. In: CONGRESSO BRASILEIRO DE ENFERMAGEM OBSTÉTRICA E NEONATAL, 1, São Paulo, 1994. Anais. São Paulo, 1994.

03. CARPENITO, L.J. Nursing diagnosis: application to clinical practice. 7. ed. New York: Lippincott, 1997.

04.COREN. CONSELHO REGIONAL DE ENFERMAGEM DE SÃO PAULO. Documentos básicos de Enfermagem. São Paulo: Coren, 1997. p.101.

05. COSTA, C.A.R.; BARROS, S.M.O. Anemia e Gravidez: intervenções de enfermagem para a sua prevenção e tratamento. In: CONGRESSO PIBIC/CNPq DA UNIFESP, 4, São Paulo, 14-18 de outubro de 1996. Anais. São Paulo, 1996.

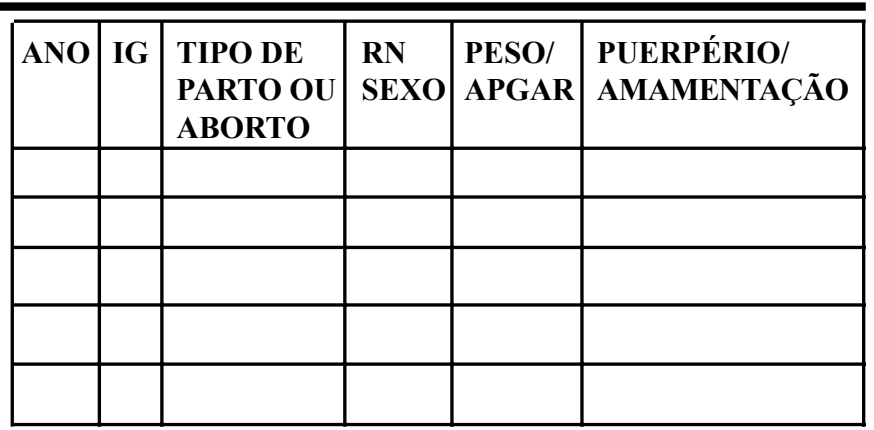

Exame físico

Sinais vitais: $\mathrm{PA}$

Hemoglobina:

Pulso F.C. F.R.

Hematócrito:

Ferro Sérico: Data:

Observações:

Conjuntivas e mucosas: ( ) Corada ( ) Descorada

Tonus muscular: ( ) firme ( ) enfraquecido

Cabelos: ( ) Secos ( ) Quebradiços ( ) Sem brilho ( ) Sedosos ( ) Com brilho

Pele: ( ) Cianótica ( ) Pálida ( ) Seca ( ) Envelhecimento precoce

Peristaltismo: ( ) aumentado ( ) diminuído

Boca:( ) Halitose ( ) Com lesão ( ) Sem alterações

Língua: ( ) Lisa ( ) Quebradiço ( ) Descamação ( )

Saburrosa

Dentes:( ) Ausência ( ) Cariados ( ) Com prótese ( ) Normais

Outras Observações:

Enfermeira

COREN

06. DOENGES, M.E.; MOORHOUSE, M.F. Aplicação do processo de enfermagem e do diagnóstico de enfermagem. Lisboa, 1992. 252p

07. GALLANI, M.C.B.J.; COLOMB, R.C.R.; CINTRA, E.A.; RIGACCI, S.B. Coleta de dados: avaliação de um modelo piloto. Rev.latinoam.enfermagem, Ribeirão Preto, v. 4, n. 2, p.179-199, Julho 1996.

08. GORDON, M. Nursing diagnosis, process and application.3. ed. New York: Mosby, 1994.

09. HASSMAN, M. Protocolo sistematizado de levantamento de dados e diagnósticos de enfermagem na Clínica Ginecológica. São Paulo, 1996. Monografia (Especialização em Enfermagem Obstétrica) - Universidade Federal de São Paulo.

10. LIMA, C.S.P.; ARRUDA, V.R.; COSTA, F.F. Hemopatias: anemias. In: NEME, B. Obstetrícia básica. São Paulo: Savier,1994. Cap. 37, p.265267.

11. MARIA, V.L.R. Preparo de enfermeiras para utilização de diagnósticos de enfermagem: relato de experiência. São Paulo, 1990. Dissertação (mestrado) - Escola de Enfermagem, Universidade de São Paulo. 
12. NANDA. North American Nursing Diagnosis Association. NANDA nursing diagnosis: definition and classification 1997-1998. Philadelphia: NANDA, 1996.

13. OMS. Organização Mundial da Saúde. Anemias nutricionais. Ginebra, 1968. (Série Informes técnicos, 405).

14. ROODENBURG, A.J.C. Iron suplementation during pregnancy. Eur.J.Gynecol. Reprod. Biol., v. 61, p. 65-71,1995.
15. SILVA, G.P. Identificação dos diagnósticos de enfermagem em puérperas, ultilizando a classificação proposta pela NORTH AMERICAN NURSING DIAGNOSIS ASSOCIATION (NANDA). São Paulo, 1995. Monografia (Especialização em Enfermagem Obstétrica) - Universidade Federal de São Paulo. 